\title{
Enhanced Situational Awareness and Decision Support for Operators of Future Distributed Power Network Architectures
}

Zaher, Ammar S. A. E.; Catterson, V. M.; Syed, M. H.; McArthur, S.D.J ; Burt, Graeme M.; Marinelli, Mattia; Prostejovsky, Alexander

\section{Published in:}

Proceedings of 2016 6th IEEE PES International Conference and Exhibition

Link to article, DOI:

10.1109/ISGTEurope.2016.7856198

Publication date:

2016

Document Version

Peer reviewed version

Link back to DTU Orbit

Citation (APA):

Zaher, A. S. A. E., Catterson, V. M., Syed, M. H., McArthur, S. D. J., Burt, G. M., Marinelli, M., \& Prostejovsky, A. (2016). Enhanced Situational Awareness and Decision Support for Operators of Future Distributed Power Network Architectures. In Proceedings of 2016 6th IEEE PES International Conference and Exhibition IEEE. https://doi.org/10.1109/ISGTEurope.2016.7856198

\section{General rights}

Copyright and moral rights for the publications made accessible in the public portal are retained by the authors and/or other copyright owners and it is a condition of accessing publications that users recognise and abide by the legal requirements associated with these rights.

- Users may download and print one copy of any publication from the public portal for the purpose of private study or research.

- You may not further distribute the material or use it for any profit-making activity or commercial gain

- You may freely distribute the URL identifying the publication in the public portal 


\title{
Enhanced Situational Awareness and Decision Support for Operators of Future Distributed Power Network Architectures
}

\author{
A. S. Zaher, V. M. Catterson, M. H. Syed, S.D.J McArthur and G. M. Burt \\ Institute for Energy and Environment, University of Strathclyde, Glasgow, Scotland \\ M. Marinelli, A. Prostejovsky \\ Centre for Electric Power and Energy, Technical University of Denmark, Roskilde, Denmark
}

\begin{abstract}
This paper describes scenarios proposed for a control room decision support system aimed at future power network operators. The purpose is to consider the requirements of the future control room from the perspective of the operator under the conditions of a significant frequency excursion incident. The control room visualisation and decision support functionality for aiding the operator in restoring the frequency to its target value will be considered. The analysis takes place within the Web-ofCells framework, adopted to deal with power system control through a web of subsystems, called cells, which are highly automated, and operated by Cell Operators.
\end{abstract}

\section{INTRODUCTION}

With the rapidly increasing levels of automation and intelligence within today's power systems, the control room of the future may look significantly different to the traditional concept of visualising the network diagram and real time alarms. On the one hand, there is ever more data being generated and collected by wide area measurement systems, phasor measurement units, and online condition monitoring, and control room operators will need additional support in order to sift and prioritise key information. On the other hand, automation systems to achieve active network management, auto-reclosing capability, and dynamic plant ratings lead to a network that is more self-managing and self-healing than ever before, reducing the burden on operators to take action.

This means that the role of the control room operator will inevitably change. The control room as a physical location may not exist, with typical operator functions being the responsibility of individuals who may either be co-located or distributed remotely. Systems to assist operators must likewise adapt and evolve to meet the needs of future engineers, while still ensuring emergency situations can be recovered when smart systems reach their limits of operation.

This paper considers one particular view of future networks, as posited by the EU project ELECTRA (European Liaison on Electricity Committed Towards Long-term Research Activity), called the Web of Cells [1,2]. In this vision, transnational grids are formed by network sections operating as peers, without hierarchical control. The operator is tasked with control of one or more cells, and incidents can be resolved by actions taken within the cell, or by requesting support from neighbouring cells.

This paper considers the particular scenario of managing a significant frequency excursion within the Web of Cells architecture, from the point of view of the control room operator managing one cell. Within this paper, "control room operator" is intended to mean the engineer with responsibility for online management of the network, and does not reflect their physical location. With reference to the literature on future control rooms, a set of requirements from the operator in this scenario is identified. The role of decision support is discussed, and the functionality in terms of prioritising appropriate actions is presented.

II. POWER SYSTEM CONTROL ROOM DECISION SUPPORT

The operation of power systems is an extremely challenging task mainly due to the size and complexity of networks and the large number of contingencies that can occur. Because of the large size and complicated nature of networks, forming a complete and accurate picture of the state of a particular area for which an operator might be responsible, can be quite difficult. These difficulties have the potential to prohibit operators from achieving the required level of situational awareness (SA) to make informed decisions and respond effectively to any particular incident.

It has been shown recently that the lack of SA has been recognised as a significant contributing factor to several large electrical disturbances worldwide, with numerous incidents including the Italian blackout in 2003 to the more recent black out in Arizona and South Carolina in 2011 [3]. It is thought that one of the main reasons for the escalation of incidents is due to poor information sharing between operators, and or failure in the information systems, leading to delayed and inadequate responses being taken [3].

Decision Support Systems (DSS) have been incorporated in power system control rooms in order to help operators 
avoid such situations. The concept of DSS was derived from the combination of theoretical studies of organizational decision making at the Carnegie Institute of Technology and technical computing advances from the 1950s onwards [4]. It was from this point that DSS grew to a distinct research topic.

A DSS can be generically defined as an information system that is designed to support business or organisational decision making activities [4]. Such a system typically serves the management and operation or planning levels in an organisation with the aim of supporting users to make decisions by providing the right information at the right time.

DSSs have been applied to a variety of applications within the power systems domain. Vale et al [5] detail a DSS to help operators with demand response and contracts adoption. This system uses machine-learning techniques to create a rule base, which describes the actions of different actors in demand response programs. A number of works exist in the area of intelligent alarm processing from different authors looking at prioritising, suppressing, and fault diagnosis of generated alarms [6-9]. The main objective of these systems is to filter the amount of data presented to the operator so that the highest priority information is passed to them.

Research in this area of DSS has more recently fallen under the wider topic of situational awareness (SA), which encompasses a more holistic view of how to improve operator awareness through a more complete assessment of the network and its ICT systems. One definition of SA has been expressed as: the perception of the elements in the environment within a volume of time and space, the comprehension of their meaning and a projection of their status in the near future [3]. Aspects such as optimised PMU placement [10] and improved GUI visualisations [11-13] as well as the alarm processing research mentioned previously all fall under situational awareness.

\section{FUTURE DistRIBUTED POWER NETWORK}

\section{ARCHITECTURES}

One vision of the power system in 2030 and beyond is for the network architecture to correspond to a Web of Cells. The Web of Cells was conceived as a means of achieving distributed control among autonomous regions of the network, termed cells [1]. A cell is defined as "a group of interconnected loads, distributed energy resources and storage units within well-defined grid boundaries corresponding to a physical portion of the grid and to a confined geographical area" [1]. The key feature is that each cell is a peer of other cells, with no overall system operator able to exercise hierarchical control over cells. It is expected that peer cells will collaborate to efficiently allocate resources, including power and ancillary services, via tie lines between cells [2].

Trends within power systems are such that distribution networks are becoming more like traditional transmission systems, with bidirectional power flows, more active control, and greater observability than in the past. This trend is expected to continue, so that by 2030 there may not be a split between Transmission System Operators and Distribution System Operators, but simply Cell Operators [2]. A cell is not confined to a particular voltage level, and some cells may span voltage levels. Therefore, it is expected that current utilities will transition to operating one or more cells, which will generally correspond to their current geographical network regions.

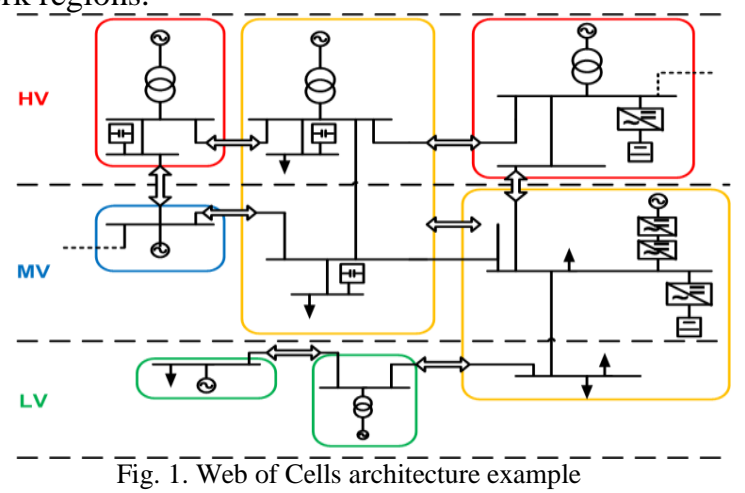

With this in mind, an operator will have certain responsibilities for managing their cell, such as maintaining voltage and thermal constraints, responding to frequency deviations, and so on. Periodically (such as on a 15-minute basis), each cell will commit to a plan of service delivery with neighbouring cells, to ensure contingencies can be handled. The operator is able to take unilateral action within their cell, as long as they continue to meet their commitments to neighbours.

This change in operator responsibilities places new requirements on the data and information systems the operator needs to access. As some examples:

- The operator needs to be able to visualise the current state of commitments to neighbours, and any constraints on satisfying them such as tie line capacity. It is expected that network automation will achieve these commitments without breaching constraints in the general case, so no operator action is required. But awareness of which constraints are reaching their limits is beneficial, as an internal cell reconfiguration instigated by the operator may alleviate congestion.

- The operator must be able to see a forecast of events within the committed time period, in order to identify looming threats to service delivery. It may be that a tie line is within capacity limits now, but a generator is scheduled to go offline with a significant impact on intercell power flows. It is easier to resolve such situations with foresight of their occurrence, rather than responding in a reactive manner as they occur. A forecast of headroom on each constrained asset and flexibility within dispatchable loads is needed to assist with this.

- The operator needs to be able to find out which automatic control systems are operating, but at the same time not be overwhelmed by notifications of every automated action within the cell. There may be times when automated systems such as active network management schemes are disabled, due to outages for maintenance or other events. The operator needs to be able to predict the outcome of manual control actions, including the response (or nonresponse) of controllers. But the high level of automation expected within a cell would be mentally taxing if events were presented like SCADA alarms. Therefore, there 
must be a way for the operator to "drill down" to the status information for automated systems.

- The operator must be able to take action if and when the automated control systems fail to resolve a contingency event. Automated systems will be designed to handle particular contingencies, such as a certain loss of generation. If the size of the event exceeds the designed capabilities of the controller, the network may not be automatically recoverable. It is expected that in extreme circumstances, automatic load shedding will be initiated as a final preservation strategy. However, the human operator may know of alternative strategies to maintain network operation, or may wish to alter the priority of loads for shedding given specific knowledge. It should be possible for the operator to override the automated systems in such a case.

These requirements highlight two broad functions for the information system: it must support visualisation of current and future state (Situational Awareness); and it must allow control actions to be taken. Since the latter is expected to occur only in unusual circumstances (such as extreme network events), it would be beneficial for the information system to also provide decision support at such times, guiding the operator as to what actions are available, and their expected outcomes.

The next section considers a particular case study of managing a frequency excursion within a Web of Cells. The role of the operator, and the functionality of the information system to support that role, are proposed and discussed.

IV. DECISION SUPPORT DURING FREQUENCY DEVIATIONS

A critical incident which any network must be prepared to handle is that of a significant frequency deviation event. This section explores how such a scenario might be handled within the Web of Cells framework. Any event that causes a significant deviation in frequency beyond the operational limits is managed by the following three services:

1. Frequency Containment Control (FCC): As the name suggests, the main responsibility of this service is to stop the rise or fall of frequency and to contain it to a pre-defined value.

2. Balance Restoration Control (BRC): Once the frequency deviation has been contained, it is the responsibility of $\mathrm{BRC}$ to restore the frequency back within the operational limits and the power exchange with other cells to the scheduled values.

3. Balance Steering Control (BSC): Although the frequency has been restored by BRC, the reserves that are utilized by BRC are fast acting and therefore may be expensive. These reserves would need to be replaced for the remainder of the scheduled period. Therefore, it is the responsibility of the BSC to replace the restoration reserves with the most economically feasible resources.

As shown in Fig. 2. there are two phases of frequency management within the Web of Cells architecture: a procurement phase and real-time operation phase. Although the services described earlier seem very similar to the services that are available to the operators today, it is the distributed procurement and real-time delivery of these services necessary within the Web of Cells paradigm that sets them apart. It is intended that the procurement of services is achieved by an algorithm distributed amongst the cells within the network (hereafter referred to as the "inter-cell algorithm"), which will fairly allocate resources to meet contingencies anticipated by each cell. The implementation of this algorithm is out of the scope of this paper, which focuses instead on the requirements and functionality of each actor within the cell.

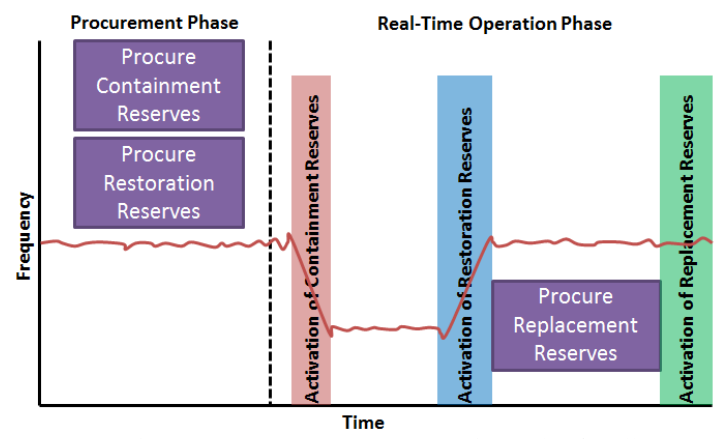

Fig. 2. Frequency management in Web of Cells

V. FUTURE CONTROL ROOM DECISION SUPPORT EXAMPLES

Two main scenarios are considered here, which fall within the category of a significant frequency excursion incident (such as the loss of two generators) within the Web of Cells. The scenarios detail how the visualisations and decision support might be provided to a cell operator under these circumstances. The two scenarios are as follows:

1. A single frequency deviation event within a cell

2. Two frequency deviation events occurring within the same cell as follows:

a. Two simultaneous or near simultaneous events

b. The second event occurs after the initial event frequency restoration phase.

For the first scenario, the automatic frequency control scheme described in the previous section is envisaged to automatically handle a single frequency disturbance event within a cell. In line with the "guidelines" detailed in [3] the authors agree that even while the system is automatically resolving the single event, key information must still be relayed to the operator so that they are kept well informed of what is happening within the network and how any particular incident is being dealt with. That is, there is no operator decision to be made, but visualisation of the situation is required. It is for this reason that the single event scenario is considered to be of interest.

The second scenario has been further split into two cases as the timing of the second event differentiates the options available to an operator when attempting to resolve the incident. Sequence diagrams have been created in order to describe the various interactions taking place between the different system actors. These actors are: 


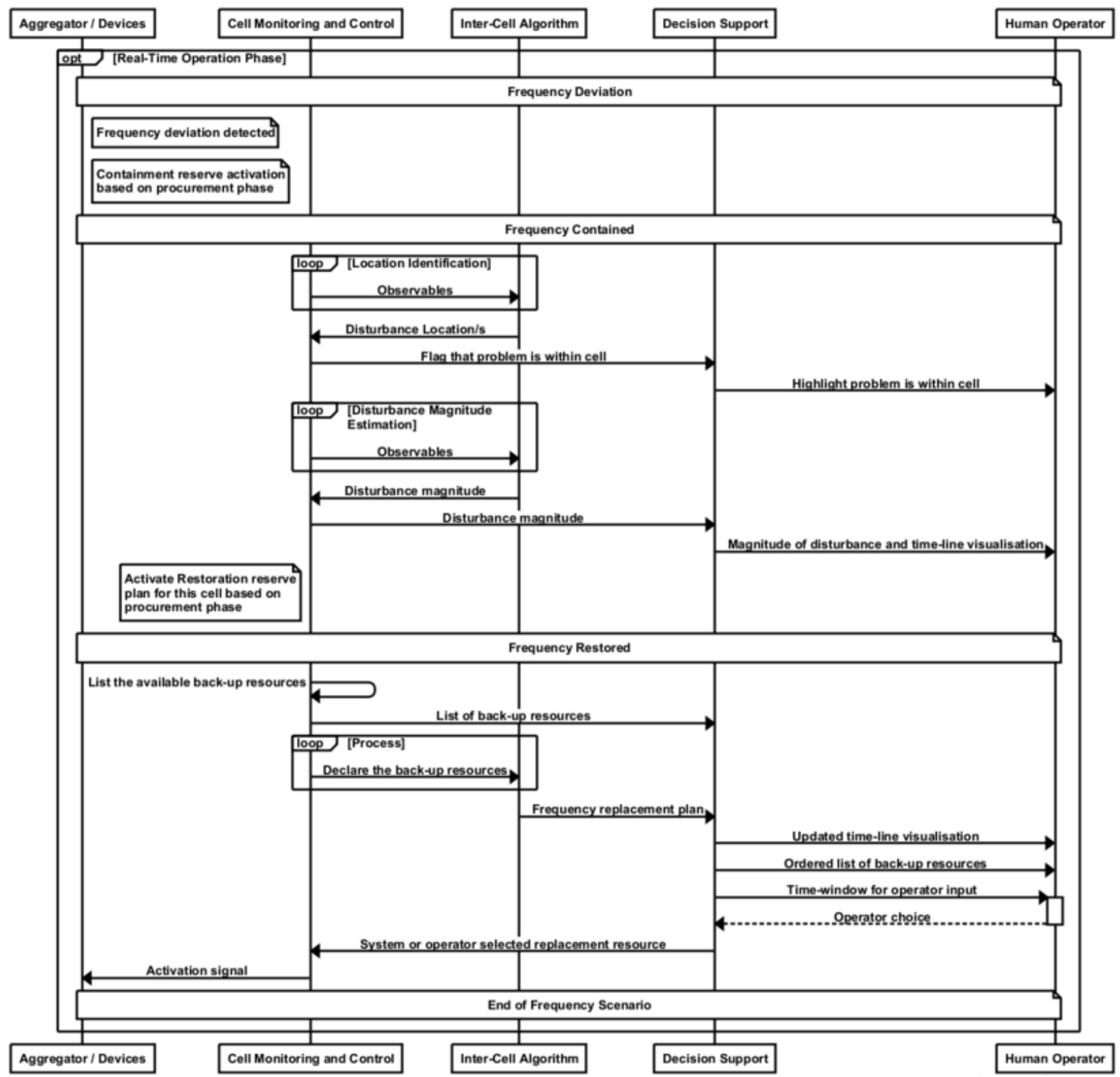

Fig. 3. Scenario 1) Single frequency event sequence diagram.

- Aggregators/devices: This refers to the controllable demand/generation that is available within the cell. In general, the aggregator is responsible for all devices that are present within their portfolio. There can be more than one aggregator within a cell.

- Cell Monitoring and Control: This is the entity within the cell that is responsible for monitoring the observables available within the network and taking control decisions. Monitoring involves the processing of all the observables and making it available to any entity as and when required. Control involves all the automated control algorithms that are present within the cell.

- Inter-cell Algorithm: This refers to the control algorithm that in a distributed manner communicates and negotiates with other cells to coordinate network restoration plans.
- Decision Support: Refers to the software system responsible for processing any available information to portray to the operator what is happening within the network. It must decide how best to show this, while aiding the operator through any processes they may need help with.

- Human Operator: is the person responsible for the secure and stable operation of the cell. Although most of the functionalities within the cell are envisioned to be automated, manual control can be given to the operator and their authority supersedes all other entities within the cell.

The aim is to explain the sequence of events as the frequency excursion incidents progress from the initial point of occurrence to finally the frequency being restored. 


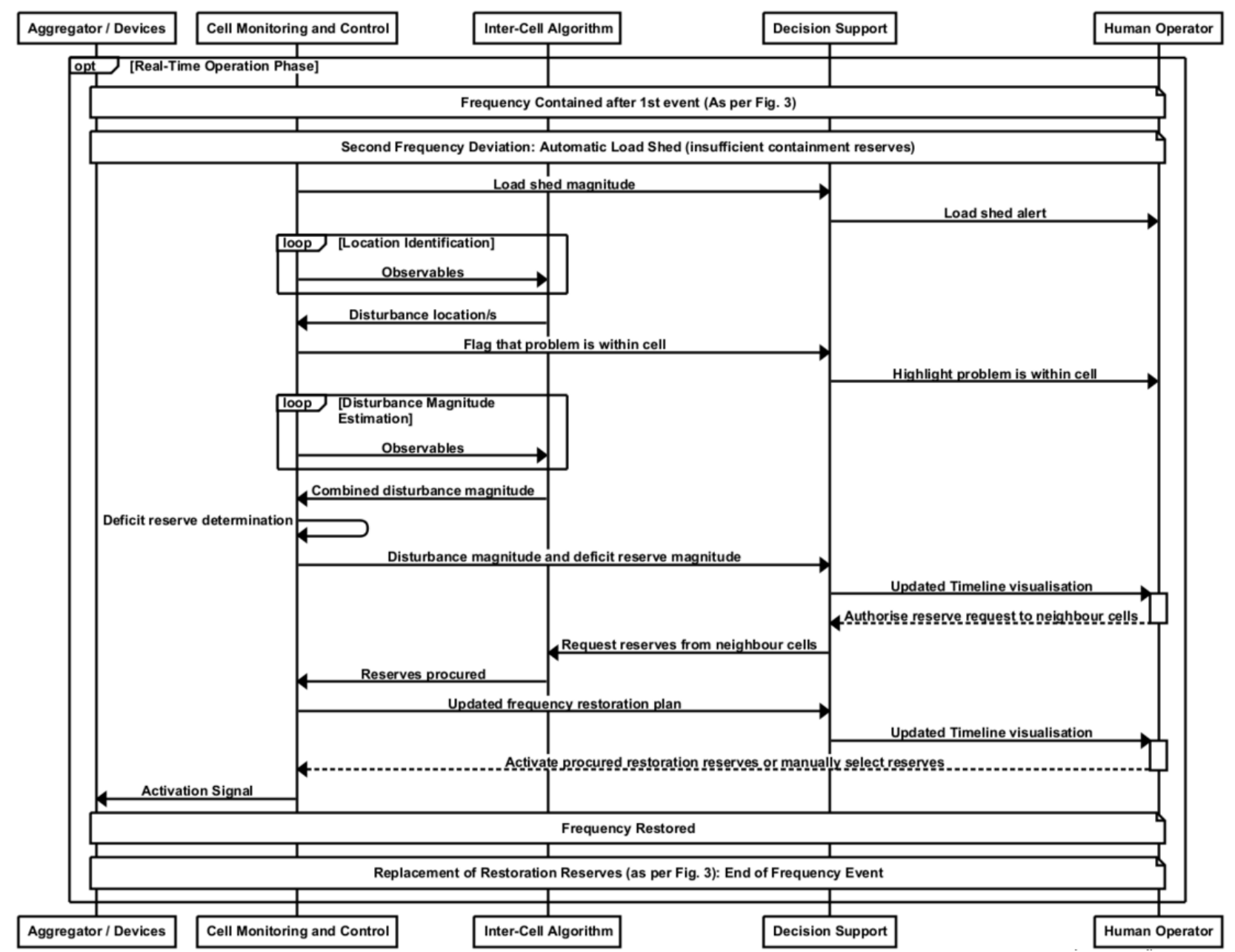

Figure 4: Scenario 2a) Two frequency events within the same cell

\section{1) Single Frequency Deviation Event}

The sequence diagrams are concerned with the real-time operation phase as shown in Figure 2. Therefore, it is assumed that at the start of each diagram, there is an agreed plan for containment reserves and restoration reserves for the current scheduled period, which has been communicated to devices as appropriate. Figure 3 details the operation phase for the single frequency event.

The process starts with the frequency deviation incident being detected locally at the cell level by the Aggregator, where the frequency is monitored. The containment reserve plans are automatically activated to contain the frequency. FCC is fast acting (within milliseconds) and so there is no time for these events to be relayed to the operator, or for the operator to alter the plan. However, the basis for the containment reserve plan is that its activation will contain a single frequency deviation event.

Following this, the cell control mechanisms attempt to identify the location of the incident to prepare for the frequency restoration phase and BSC. Measurements are taken from within the cell, and appropriate information shared with neighbouring cells.

Once this process is complete, the problem cell is identified to the operator. The information passed to the operator will differ depending on whether they operate the problem cell or not. If the problem is within a distant cell, only an indication that there is a problem in the network currently being resolved is needed, as no action from within this cell is necessary. A cell neighbouring the problem cell will display a visualisation of how their reserves are contributing to the restoration of the frequency. At this point the neighbouring cell can automatically calculate its available flexibility over and above its current commitment so that it is already preparing for a subsequent incident should that occur.

In the scenario shown in Figure 3, the problem originated within the cell of this operator. Therefore, the DSS presents updated visualisations detailing this and the activated plans for resolving the excursion to the human operator. It is envisaged that a timeline visualisation indicating the current frequency of the network and a forecast of the result of the restoration plans is displayed. This ensures that the operator is kept informed with what is happening in the network, especially if it falls within their responsibility area. Finally, for the BSC phase, a list of back-up resources available to the cell is provided to the DSS. The DSS then processes this information to prioritise the resources for the operator in terms of suitability and price. Cell control and monitoring also provides an automated frequency replacement plan, 
giving the operator the choice of implementing this or utilising a plan based on their manual selection from the prioritised list. The time-line visualisations would also be updated accordingly based on the operator selection, to reflect the new replacement reserves. The operator has a certain window of time in which to make their decision, after which if no response has been received, the decision support automatically signals the cell control to implement its plan.

It is worth mentioning here that for the single frequency event scenario, it can be seen that no actions are really necessary from an operator's point of view as everything is dealt with automatically. Nevertheless, actions can still be taken if needed to manually override the executed plans.

\section{2) a) Two simultaneous frequency events}

This second scenario starts with the same frequency containment process as before, however a second frequency event occurs almost instantly within the same cell. As there are insufficient containment reserves to deal with this excursion, automatic load shedding takes place to contain the frequency. This information is passed to the operator. The same disturbance location and magnitude identification process takes place as before, with the total combined disturbance from both events now being calculated. This allows for the deficit in the restoration plans obtained during the procurement phase to be determined and highlighted to the operator using the time-line visualisation.

As the load shed process has taken place by this point, the operator now has some time to take action and authorise a reserve request to the neighbouring cells. This can be either in the form of asking for an automated plan to be formulated by the cell control mechanisms or by manually formulating a plan by requesting reserves from the desired neighbouring cells. Once the extra reserves are procured, the full frequency restoration process can be completed and the visualisations showing the time-line of how and when the plan will restore the frequency of the network can be updated accordingly.

\section{2) b) Second event occurs after initial event restoration}

In this scenario, a second frequency event occurs after the initial restoration plans have been executed and when the frequency of the network is being restored. However, due to the second event, the frequency will not restore to its target level. No load shed takes place at this point of time as the restoration reserves have the effect of containing the frequency. From the point that the frequency is contained, the process is similar to that described for $2 \mathrm{a}$, with the operator being given the option of altering the restoration plan.

\section{CONCLUSION}

This paper has explored the requirements of a control room of 2030 , from the point of view of a cell operator within the Web of Cells architecture. With the anticipated levels of automated control within future power networks, there are many situations where no operator action will be required. However, there are ever increasing volumes of data being gathered from power networks, and operators will require tools for prioritising information, particularly during network events such as frequency deviations. This work proposes the functionality of the control room visualisation and decision support system, which can restore the frequency to its target value after such an event.

\section{ACKNOWLEDGMENT}

The work in this paper has been in part supported by the European Commission, under the FP7 project ELECTRA (grant no: 609687).

\section{REFERENCES}

[1] L. Martini, L. Radaelli, H. Brunner, C. Caerts, A. Morch, S. Hanninen, C. Tornelli, "ELECTRA IRP Approach to Voltage and Frequency Control for Future Power Systems with High DER Penetration", 23rd International Conference on Electricity Distribution (CIRED), June 2015.

[2] M. H. Syed, G. M. Burt, J. K. Kok, R. D'Hulst, "Demand Side Participation for Frequency Containment in the Web of Cells Architecture", International Symposium on Smart Electric Distribution Systems and Technologies (EDST), September 2015.

[3] Mathaios Panteli, Daniel S. Kirschen, "Situation awareness in power systems: Theory, challenges and applications", Electric Power Systems Research, Volume 122, May 2015, Pages 140-151.

[4] Keen, P. G. W. (1978). "Decision support systems: an organizational perspective.” Reading, Mass., Addison-Wesley Pub. Co.

[5] Faria, Pedro, and Zita Vale. "Decision Support Concerning Demand Response Programs Design and Use-A Conceptual Framework and Simulation Tool." Applied Mathematics \& Information Sciences 8.1 (2014): 161.

[6] G. M. Burt, J. R. McDonald, A. G. King, J. Spiller, D. Brooke and R. Samwell, "A real-time decision support system for the operation of a $132 \mathrm{kV}$ power network," Control, International Conference on, Coventry, UK, 1994, pp. 153-158 vol.1.

[7] R. W. Bijoch, S. H. Harris, T. L. Volkmann, J. J. Bann and B. F. Wollenberg, "Development and implementation of the NSP intelligent alarm processor for power systems," in IEEE Transactions on Power Systems, vol. 6, no. 2, pp. 806-812, May 1991.

[8] Alvin H. Shoop, Steven Silverman, "A real-time alarm processor", International Journal of Electrical Power \& Energy Systems, Volume 14, Issue 2, 1992, Pages 108-113.

[9] E. Kyriakides, J.W. Stahlhut, G.T. Heydt, "A next generation alarm processing algorithm incorporating recommendations and decisions on wide area control", In: Proc. of the IEEE Power Engineering Society General Meeting, 2007.

[10] R. Emami and A. Abur, "Robust Measurement Design by Placing Synchronized Phasor Measurements on Network Branches," in IEEE Transactions on Power Systems, vol. 25, no. 1, pp. 38-43, Feb. 2010.

[11] T.J. Overbye, D.A. Wiegmann, A.M. Rich, Y. Sun, "Human factors aspects of power system voltage contour visualizations", in: Proc. of the IEEE Power Engineering Society General Meeting, 2003.

[12] R.P. Klump, J.D. Weber, "Real-time data retrieval and new visualization techniques for the energy industry", in: Proc. of the 35th Annual Hawaii International Conference on System Sciences (HICSS), 2002.

[13] T.J. Overbye, J.D. Weber, "Visualization of power system data", in: Proc. of the 33rdAnnual Hawaii International Conference on System Sciences (HICSS), 2000. 